Betätigungen teil und verbringen mehr Zeit vor dem Fernseher und Computer.

Hanć T et al. Attention-deficit/hyperactivity disorder is related to decreased weight in the preschool period and to increased rate of overweight in school-age boys. J Child Adolesc

Psychopharmacol 2015;25:691-700

\section{Kommentar}

Diese Studie bestätigt die klinischen Beobachtungen:

1. Das Längenwachstum ist bei Kindern mit ADHS verzögert, unabhängig von einer medikamentösen Therapie. Diskutiert wird hier eine konstitutionelle Entwicklungsverzögerung.

2. Ein Großteil der Kinder mit ADHS ist nicht nur kleiner, sondern auch untergewichtiger - jedenfalls im Vorschulalter.
3. Ab einem gewissen Alter verschwindet das Phänonem des Untergewichts jedoch: Adipositas tritt auf.

Welche Einflüsse im Alter von etwa 8 Jahren bei den Kindern mit ADHS zu einer deutlichen Gewichtszunahme führen, bleibt unklar. Die Autoren konnten den sozialen Status, das Geburtsgewicht und Frühgeburtlichkeit - alles Faktoren, die immer wieder im Zusammenhang mit Adipositas diskutiert werden - als Ursache der Adipositas bei Kindern mit ADHS ausschließen. Auch ein möglicher Reboundeffekt einer medikamentösen Therapie ist ausgeschlossen, da bei den Kindern noch keine Therapie gestartet wurde. Mögliche Ursachen sind impulsives Essverhalten bei nachlassendem Kalorienverbrauch (motorische Hyperaktivität nimmt ab) oder auch Folge von sozialem Außenseitertum mit Frustessen. Interessant wäre hier eine Verlaufsstudie des Gewichts der Kinder mit ADHS ab dem Alter von 8 Jahren jetzt unter Medikation. Aus der Metaanalyse von Cortese et al. (vgl. Beitrag auf Seite 15) wissen wir, dass die Adipositas bei Menschen mit ADHS kein vorübergehendes Phänomen, sondern auch im Erwachsenenalter noch anzutreffen ist.

Der Einfluss einer Adipositas auf die Gesundheit, aber auch auf die soziale Akzeptanz, sollte nicht übersehen werden. Auch wenn die Therapie einer Adipositas keine Indikation für die Stimulanzientherapie ist, so sollte bei Kindern mit ADHS, vor allem wenn zusätzlich eine Adipositas vorliegt, der Einsatz einer Therapie mit Methylphenidat erwogen werden. Dr. Kirsten Stollhoff

\title{
AWMF-Leitlinie zur venösen Thromboembolie revisionsbedürftig?
}

Trotz des Wissens um die Bedeutung der Nierenfunktion bei der Behandlung einer venösen Thromboembolie sind Prävalenzen kaum bekannt. In einer aktuellen Arbeit aus der Erwachsenenmedizin wird die Leitlinien-Therapie mittels unfraktionierter Heparine überprüft.

m Kindesalter treten Thrombosen am häufigsten in der Neugeborenenzeit und zu Beginn der Pubertät auf (Abb. 1). Zur Antikoagulation bei Patienten mit venöser Thromboembolie (VTE) empfehlen die Leitlinien unfraktionierte Heparine (UFH).

Zur Überprüfung der leitlinienbasierten Therapie mit unfraktionierten Heparinen wurde eine systematischen Daten- banksuche von Publikationen aus den Jahren 2004-2014 durchgeführt. Es konnten 1.135 Studien identifiziert werden, von denen 37 die Kriterien zur Auswertung erfüllten.

Überraschend zeigte keine der ausgewerteten Studien Effektivitäts- oder Sicherheitsvorteile der UFH im Vergleich zu niedermolekularen Heparinen (NMH) bei VTE-Patienten mit stark ein-

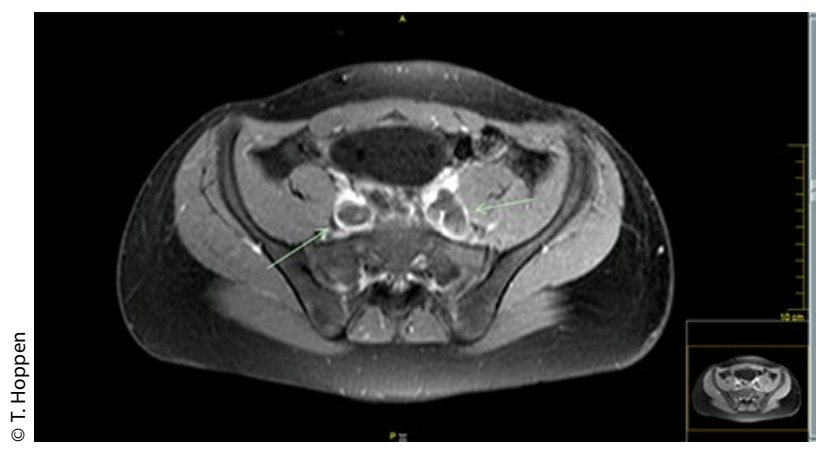

Abb.1: Abdomen-MRT einer 16-jährigen Patientin mit einer ausgedehnten Thrombosierung der linksseitigen Femoral-, Becken- sowie Lumbalvenen und der Lumbalvenen rechts von der Vena iliaca communis bis Höhe LWK 4 bei Aplasie der Vena cava inferior geschränkter Nierenfunktion. Es scheint sogar, dass die UFH-Behandlung unterlegen ist.

Die Autoren der deutschen Studie legen eine kritische Prüfung der aktuellen AWMF-Leitlinie nahe. Die Empfehlung von UFH als Therapie der ersten Wahl bei Niereninsuffizienz erscheint somit revisionsbedürftig.

Wilke T et al. Renal impairment in patients with thromboembolic event: prevalence and clinical implications. A systematic review of the literature. Dtsch Med Wochenschr 2015;140:e166-74

\section{Kommentar}

Bei Erwachsenen haben 5-7\% der initial behandelten Patienten mit venöser Thromboembolie eine stark eingeschränkte Nierenfunktion (Kreatinin-Clearance $<30 \mathrm{ml} /$ min). Für diese Patientengruppe bedarf es einer besonders vorsichtigen Auswahl und Dosierung der antikoagulierenden Therapie. Neben den möglicherweise kritisch zu prüfenden Angaben der aktuellen AWMFLeitlinie sollte man mit den Fachinformationen der Substanzen vertraut sein. Auf Grundlage der Empfehlungen zu Dosierungsschemata, Kontraindikationen bei eingeschränkter Niereninsuffizienz und natürlich für Heranwachsende immer grundsätzliches Problem - dem Zulassungsalter sollte die Therapiewahl erfolgen. Dr. Thomas Hoppen 